\title{
Le jugement professionnel en évaluation: un acte cognitif et une pratique sociale située
}

\section{Lucie Mottier Lopez et Linda Allal}

L'article examine les caractéristiques du jugement professionnel en évaluation par les enseignants, conceptualisé comme étant un processus cognitif et une pratique sociale liée à différents contextes. La multiréférentialité du jugement professionnel en évaluation est argumentée, montrant que celui-ci convoque des cadres de référence et des repères d'ordres différents. L'article présente une recherche sur les démarches d'évaluation sommative et pronostique mises en æuvre par une dizaine d'enseignants genevois de Gème primaire. Les résultats décrivent les procédures utilisées par les enseignants pour établir les notes dans le livret scolaire et les ajustements évaluatifs quils effectuent au regard de situations singulières d'élèves. Les dimensions sociales et éthiques du jugement professionnel en évaluation sont soulignées. Une modélisation en quatre points est esquissée. Elle peut fonder des activités de formation et de collaboration collégiale aptes à développer la cohérence et la rigueur du jugement professionnel des enseignants et à limiter ainsi les biais affectant leurs pratiques de notation.

Cet article expose une perspective qui s'éloigne de l'idée que l'évaluation consisterait à situer mécaniquement les élèves sur des échelles de mesure sans prise en compte des pratiques et des significations sociales et culturelles dans lesquelles s'enracinent les décisions évaluatives. Dans cette perspective, nous examinons plus spécialement le jugement professionnel des enseignants en matière d'évaluation scolaire. Lafortune (2006) définit le jugement professionnel de l'enseignant comme:

un processus qui mène à une prise de décisions, laquelle prend en compte différentes considérations issues de son expertise (expérience et formation) professionnelle. Ce processus exige rigueur, cohérence et transparence. En ce sens, il suppose la collecte d'informations à l'aide de différents moyens, la justification du choix des moyens en lien avec les visées ou intentions et le partage des résultats de la démarche dans une perspective de régulation (p. 22).

Cette définition du jugement professionnel n'est pas propre à l'évaluation, mais concerne l'ensemble des activités de l'enseignant, notamment le choix et l'agen- 
cement des situations didactiques, la gestion de la classe et des activités d'enseignement, les échanges avec les collègues sur des projets communs, ainsi que toutes les étapes de l'évaluation des apprentissages qu'elle soit formative, sommative ou pronostique (Allal \& Lafortune, 2008). Autrement dit, le jugement professionnel n'est pas restreint à un aspect particulier du processus évaluatif .

Dans cet article, notre propos porte sur des situations d'évaluation dites sommatives, c'est-à-dire qui contribuent à la reconnaissance des compétences et à la certification des apprentissages des élèves, et sur des évaluations dites pronostiques qui servent à fonder les décisions à prendre à propos de l'avenir des élèves. Par rapport aux différents travaux qui ont étudié le jugement évaluatif (voir l'article de Laveault, dans ce numéro), notre but est d'examiner les caractéristiques de ce jugement lorsqu'il relève de la professionnalité de l'enseignant. Sans entrer dans le vaste débat sur le statut plus ou moins controversé de l'enseignant-professionnel, nous examinons la façon dont le jugement en évaluation se formule au regard de quelques conditions propres à la profession enseignante (e.g., Gohier, Bednarz, Gaudreau, Pallascio \& Parent, 1999; Lafortune \& Allal, 2008; Laveault, 2005; Paquay, Altet, Charlier \& Perrenoud, 1996), notamment lorsque

- le jugement évaluatif s'exerce dans des situations de problèmes complexes pour lesquelles l'application de règles standardisées ne convient pas,

- la résolution nécessite des savoirs d'expérience et la mobilisation de connaissances professionnelles,

- des prises de décision qui relèvent de la responsabilité et d'une certaine autonomie de l'enseignant,

- une analyse des ressources et des contraintes liées aux contextes concernés est impliquée,

- une réflexion critique tant individuelle que collective est suscitée,

- des formes d'ajustements créatifs au regard des situations singulières sont engagées.

Le jugement professionnel ne s'exerce pas seul; il se réalise en interaction plus ou moins directe avec les personnes impliquées par les décisions qui en résultent (l'enfant et sa famille), avec le groupe professionnel de proximité de l'enseignant (ses collègues et autres professionnels avec qui il travaille) et toujours dans une relation à l'institution scolaire et plus largement à la société. Ce faisant, le jugement professionnel en évaluation s'inscrit dans une multiréférentialité (Mottier Lopez, 2007, à paraître) qui implique des cadres de référence et des repères d'ordres différents: les normes professionnelles perçues comme reconnues et partagées par les acteurs; les exigences institutionnelles formalisées dans les lois, règlements ou principes d'application; les choix collégiaux d'une école ou d'un établissement qui parfois se concrétisent dans une charte; la microculture de la classe qui est construite entre chaque enseignant et ses élèves délimitant les attentes, les normes, les pratiques partagées au regard des activités effectivement réalisées; les valeurs personnelles de l'enseignant et ses croyances qui intègrent, 
entre autres, les aspects plus ou moins conscients du jugement évaluatif (Laveault, 2005). Comme souligné par Allal et Lafortune (2008), la complexité du jugement professionnel résulte notamment des décalages, voire des divergences entre ces différents repères, et aussi entre les différentes informations recueillies et interprétées à propos d'un même élève. De manière générale et surtout en situation d'incertitude, le jugement professionnel fonde sa légitimité sur les principes déontologiques et éthiques de la profession enseignante. ${ }^{1}$

Dans cet article, nous nous appuierons sur les résultats d'une recherche que nous avons menée auprès d'une dizaine d'enseignants genevois de sixième année primaire. ${ }^{2}$ Cette recherche visait à examiner les caractéristiques du jugement professionnel dans des situations d'évaluation sommative et pronostique étudiées à travers des entretiens avec les enseignants et sur la base des documents évaluatifs qu'ils établissent (indications inscrites dans le livret scolaire, instruments d'évaluation, registres de résultats, traces d'observations, etc.). Nous mettons en relation les principaux résultats de deux volets de la recherche, à savoir:

- les procédures élaborées par l'enseignant pour attribuer les notes en fin de trimestre et d'année (Mottier Lopez \& Cattafi, 2008);

- les ajustements évaluatifs introduits par l'enseignant au regard des situations singulières de certains élèves et les démarches de communication qui entourent les notes (Allal \& Hohl, 2008).

Notre postulat est que le jugement de l'enseignant doit être problématisé comme un processus cognitif individuel (mise en relation d'informations diverses, raisonnement, interprétation, appréciation, prise de décision) et tout à la fois comme une pratique sociale marquée par les contextes sociaux et culturels dans lesquelles il se situe (concertations avec des collègues, échanges avec les parents d'élèves, entre autres) et/ou qu'il convoque plus ou moins directement (référence aux consignes et directives de l'institution scolaire, au code déontologique de la profession, notamment). L'enjeu est donc de mettre en lumière, à partir des résultats de notre recherche, les dimensions à la fois individuelles et sociales du jugement professionnel en évaluation que nous allons qualifier de «situé», afin, d'une part, de tenter de mieux comprendre les processus qui le constituent et, d'autre part, d'illustrer en quoi ce jugement de l'enseignant peut être vu comme relevant de sa professionnalité.

\section{Le jugement professionnel en évaluation "située"}

La perspective située (fondée sur les théories de l'apprentissage et de la cognition situés, e.g., Brown, Collins \& Duguid, 1989; Lave \& Wenger, 199133) engage à accorder une importance particulière aux contextes de médiation de l'activité évaluative en termes de normes, de pratiques, de significations, de discours, d'outils vus comme socialement reconnus et partagés par une ou des " commu- 
nautés de pratique». Celles-ci fournissent un cadre interprétatif qui donne sens aux activités et aux connaissances de ses membres tout en étant constitutives des pratiques sociales et des apprentissages réalisés.

À partir de ce point de vue théorique, la conception d'une évaluation située demande que celle-ci soit conceptualisée dans ses relations dialectiques avec les communautés socioculturelles dans lesquelles et par lesquelles elle se constitue, se négocie, se comprend. L'évaluation devient dès lors foncièrement interprétative, non seulement par rapport à des référentiels «classiques» de l'évaluation (institués dans les plans d'études, puis tel qu'effectivement (re)définis et utilisés par les praticiens ${ }^{4}$ ) mais également en considérant les pratiques, les valeurs, les normes souvent implicites des communautés de pratique (Mottier Lopez, 2007). Les démarches d'interprétation au cœur de l'évaluation sont fondatrices des régulations intervenant dans les processus, les dispositifs et les cursus de formation (Allal, 2007). La triangulation des repères et des sources d'information est vue comme garante d'une plus grande validité et fiabilité des jugements interprétatifs portés (Allal, à paraître).

La perspective d'évaluation située incite à conceptualiser la relation entre l'individu-en-activité (membres qui évaluent/qui sont évalués) et le contexte de développement de cette activité, tant dans l'idée d'un contexte immédiat (la classe, l'école) que dans une relation à un contexte socioculturel, économique et historique plus large (le système scolaire, ses traditions, son rapport au marché du travail). Cette conception nous amène à étudier le jugement professionnel en évaluation comme une pratique sociale, mobilisant des gestes cognitifs qui sont foncièrement marqués par différents contextes socioculturels inter-reliés, dont les principaux sont la classe, l'établissement et l'institution scolaires. Comment l'enseignant exerce-t-il son jugement en évaluation, en tant que processus cognitif individuel et pratique sociale liée à des communautés de pratique dans et par lesquelles il se développe? Cette question générale nous conduit à interroger les prises de décisions évaluatives au regard des différentes informations recueillies par l'enseignant (résultats des élèves à des évaluations formelles, informations sur la situation plus générale de l'enfant, progression d'apprentissage en cours d'année, etc.), des normes déontologiques et éthiques de la profession enseignante, des réseaux de pratique et de communication entre les personnes impliquées, des dispositions réglementaires instituées par le système scolaire concerné.

\section{La recherche}

Notre recherche ${ }^{5}$, réalisée au printemps 2006, concerne les pratiques d'évaluation sommative et pronostique d'enseignants de $6^{\mathrm{e}}$ année primaire, soit la dernière année avant le passage des élèves dans l'enseignement secondaire. Le livret scolaire utilisé, transmis aux parents à la fin de chaque trimestre, comprend les notes chiffrées et appréciations attribuées pour les différentes disciplines sco- 
laires, les appréciations concernant la vie scolaire, les commentaires éventuels rédigés par l'enseignant et, au $3^{\mathrm{e}}$ trimestre, les résultats aux épreuves cantonales (évaluations externes). Les notes chiffrées (notes entières de 1 à 6) correspondent à des catégories d'appréciation qualitatives, définies en termes de degré d'atteinte des objectifs. Il est important de préciser que notre étude a été réalisée avant l'introduction, en septembre 2007, de nouvelles dispositions règlementaires dans l'enseignement primaire genevois qui exigent d'attribuer des notes chiffrées aux contrôles écrits ponctuels et, surtout, d'établir les notes dans le livret par le calcul de moyennes. Cela signifie que les enseignants de notre recherche pouvaient exercer leur jugement professionnel avec plus d'autonomie et recourir à des procédures plus variées et plus qualitatives que c'est le cas après 2007.

L'échantillon est composé de 10 enseignants ayant de 6 à 40 années d'expérience professionnelle. La moitié d'entre eux enseignaient dans des écoles de quartiers relativement défavorisés du canton de Genève et l'autre moitié dans des quartiers au profil inverse. Nous avons mené deux entretiens de recherche avec chaque enseignant: un, au mois de mars (fin du $2^{\mathrm{e}}$ trimestre) et un autre, en juin (fin du $3^{e}$ trimestre et aussi de la dernière année de l'école primaire). En vue de l'entretien, les enseignants devaient réunir les informations dont ils avaient tenu compte pour remplir le livret scolaire de deux élèves en particulier, pour lesquels ils avaient hésité entre la note 4 («objectifs atteints») et la note 3 («objectifs presque atteints»). Notre postulat était que le jugement professionnel devrait se manifester plus nettement dans ce type de situation complexe au regard, entre autres, des enjeux importants de promotion et d'orientation vers les filières de l'enseignement secondaire ${ }^{6}$ en fin de $6^{\mathrm{e}}$ année primaire. Les échanges ont porté sur deux disciplines différentes: les mathématiques, discipline scolaire de passage intervenant dans les décisions de promotion et d'orientation, et l'environnement (géographie, histoire, sciences naturelles), discipline pour laquelle aucune exigence n'est fixée pour la promotion et l'orientation.

Un guide d'entretien a structuré les échanges, incitant les enseignants à s'exprimer sur les différentes sources d'informations qu'ils ont utilisées pour attribuer les notes dans le livret scolaire, sur les démarches mises en œuvre pour décider de cette note, sur les modalités de communication qui accompagnent la remise du livret scolaire, sur les décisions de promotion et les conseils d'orientation. Pour toutes ces rubriques, des explications et justifications ont été sollicitées afin de mieux cerner le rôle du jugement professionnel dans les pratiques et les conceptions de l'enseignant en matière d'évaluation. En plus du discours des enseignants (enregistré puis retranscrit et codé), nous avons recueilli les traces écrites du côté des élèves (exemples de contrôles écrits, pages des livrets scolaires) et de l'enseignant (feuilles récapitulatives des appréciations, registres, cahier d'observations, etc.).

Nous avons procédé à une analyse qualitative des données récoltées. À des fins de condensation et de catégorisation, nous avons créé des "formats» de dépouillement, au sens de Huberman et Miles (1991), et nous avons établi divers 
tableaux et schémas de mise en relation des données. Nous avons également analysé les explications exprimées par les enseignants au sujet de leurs démarches d'évaluation, par rapport à plusieurs repères: références officielles, choix collégiaux, stratégies pédagogiques, valeurs personnelles, contraintes d'enseignement, soutien familial de l'élève. Nous avons tenté de rendre compte, dans notre interprétation, non pas d'un raisonnement figé par des catégories disjointes, mais d'appréhender la multidimensionnalité des justifications associées au jugement professionnel. Soulignons, pour conclure cette partie, que l'étude finale des dimensions constitutives du jugement professionnel a impliqué un croisement méthodologique entre les différentes pièces de nos dépouillements et analyses, nécessitant des va-et-vient structurés entre verbatim codés, formats, traces authentiques.

\section{Résultats}

En vue d'examiner les dimensions individuelles et sociales constitutives du jugement professionnel en évaluation, il est nécessaire de commencer par décrire les pratiques des enseignants: comment procèdent-ils à partir des évaluations ponctuelles réalisées au cours d'une période de formation donnée pour décider de la note à inscrire dans le livret scolaire? 7

\section{Des sources d'évaluation à la note dans le livret scolaire}

Comme déjà observé dans l'étude d'Allal en 1988, les enseignants de notre recherche se fondent principalement sur des "contrôles écrits ${ }^{8}$ » pour attribuer la note dans le livret scolaire, à l'exception d'un seul enseignant qui dit prendre en compte certaines activités d'apprentissage au même titre que les contrôles écrits et autres interrogations. Ce sont donc, en général, des évaluations formelles et ponctuelles qui représentent la source principale d'informations pour l'évaluation sommative et pronostique. L'analyse du discours des enseignants, mais également des traces écrites de leurs contrôles, montre que tous définissent un cadre de référence critériée pour apprécier les productions des élèves. Les objectifs d'apprentissage et les critères d'évaluation, préalablement définis, sont explicités dans un "en-tête» au début des contrôles écrits, conformément aux recommandations institutionnelles de l'enseignement primaire genevois. Ces informations sont ainsi clairement communiquées aux élèves et à leurs parents.

Sur les dix enseignants de notre recherche, sept optent, dans les contrôles écrits, pour une appréciation quantitative avec un comptage de points, et trois préferent une appréciation qualitative exprimée par des catégories verbales, des symboles, des commentaires. Les résultats sont exprimés soit sous forme de profil critérié, soit par une appréciation globale non chiffrée. Pour attribuer la note dans le livret scolaire, le geste consiste ensuite à confronter les résultats ponctuels 
produits au cours d'une période de formation donnée, et à leur donner un sens au regard de la signification des définitions officielles des notes trimestrielles.

Nous avons examiné tout spécialement la façon dont les enseignants «combinent» les différentes appréciations ponctuelles pour parvenir à la note finale. Sans entrer dans le détail des différents profils de pratique que nous avons dégagés dans Mottier Lopez et Cattafi (2008), il ressort qu'un seul enseignant transforme les appréciations ponctuelles en notes chiffrées afin de calculer une moyenne au dixième. Cet enseignant décide ensuite d'arrondir à la note supérieure ou inférieure en fonction d'informations complémentaires qu'il possède pour chaque élève. Tous les autres enseignants disent ne pas avoir besoin de recourir à un algorithme mathématique pour décider de la note. Huit d'entre eux expliquent qu'ils examinent attentivement les résultats de l'ensemble des contrôles écrits et petits tests (souvent retranscrits dans un registre ou sur une feuille récapitulative en regard des objectifs d'apprentissage). Des systèmes de traces témoignent de l'interprétation des résultats consignés: des symboles pour souligner un résultat insuffisant à un contrôle écrit, des annotations diverses, le relevé des notes trimestrielles précédentes, l'indication de la progression d'un élève, un résumé succinct d'observations, etc. C'est à partir de cet examen principalement qualitatif $^{9}$ des informations concernant chaque élève que les enseignants se font une première idée de la note trimestrielle, ou de la fourchette des notes à considérer. Un dernier enseignant, celui qui ne considère pas les contrôles écrits comme devant être forcément la source principale, explique qu'il commence par se construire une représentation globale du travail scolaire de l'élève. Il l'objective ensuite par l'examen de diverses sources d'information (contrôles écrits et activités d'apprentissage) qui l'amène à préciser la note trimestrielle à envisager.

En cas d'hésitation entre les notes 3 et 4 due à la variation des résultats de l'élève, il ressort que tous les enseignants de notre recherche introduisent des adaptations ou des ajustements de leurs procédures évaluatives qui leur permettent de considérer de façon plus différenciée les situations singulières des élèves concernés. Nous considérons que ces adaptations sont spécialement représentatives de l'exercice du jugement professionnel de l'enseignant:

La capacité de l'enseignant d'adapter son propre système d'évaluation en tenant compte des différences entre élèves, de rompre avec ses routines ou de déroger à ses propres règles afin de prendre la décision la plus adéquate possible, est un indicateur clé de posture professionnelle. (Allal \& Hohl, 2008, p. 189)

L'analyse du discours des enseignants montre que ces derniers entreprennent un examen approfondi des sources d'informations initiales pour les élèves concernés: vérification fine des objectifs atteints et non atteints dans les contrôles écrits, importance et nature des erreurs, notamment. Tous les enseignants disent aussi consulter ou se référer à des sources d'informations complémentaires, de différents ordres, soit liées: 
- aux activités d'apprentissage en classe (prise en compte, par exemple, d'activités non évaluées dans les contrôles écrits, comme des résolutions de problèmes ouverts en mathématiques, ou considération de l'étayage plus ou moins important que l'enseignant doit fournir à l'élève pour réaliser la tâche);

- à l'investissement de l'élève dans les activités scolaires (implication, participation, qualité de compréhension manifestée);

- aux progressions d'apprentissage en cours d'année, voire au cours du cycle (5e-6e années);

- aux résultats aux "épreuves cantonales», c'est-à-dire aux évaluations externes passées au dernier trimestre de $6^{\mathrm{e}}$ primaire et qui fournissent des repères sur les attentes institutionnelles pour tous les élèves du canton.

En plus de ces différents éléments, une source complémentaire importante citée par les enseignants concerne les avis et les éclairages apportés par d'autres professionnels: un enseignant d'appui ou un collègue qui a eu l'occasion d'observer l'élève dans une activité de décloisonnement ${ }^{10}$, l'ensemble des collègues d'une école ou d'un cycle dans le cadre d'un suivi collégial ${ }^{11}$, des logopédistes ou psychologues scolaires. De même, les entretiens avec les familles apportent souvent des informations éclairantes qui aident à préciser la note trimestrielle reflétant le mieux les compétences de l'élève.

Ces premiers éléments de description mettent en évidence les processus cognitifs que sollicite toute évaluation des apprentissages des élèves: donner du sens à un ensemble d'informations recueillies, les interpréter, les confronter, leur attribuer une "valeur» en vue de prendre une décision. Ces gestes cognitifs sont instrumentés par des outils d'évaluation, des cadres de référence critériée, des catégories d'appréciation, des grilles de synthèse, etc., qui structurent des procédures systématiques, communes à tous les élèves. En cas d'hésitation ou de doute, des ajustements évaluatifs se mettent en place, impliquant notamment un recueil d'informations complémentaires et un examen plus approfondi de la situation singulière de l'élève. Ces ajustements engagent une analyse critique de la situation, souvent dans une double perspective temporelle: les progrès réalisés par l'élève pendant le trimestre ou au cours de l'année scolaire écoulée (évaluation sommative), et le parcours de formation tel qu'il est projeté dans l'enseignement secondaire (évaluation pronostique). Dans les cas d'hésitation, nos analyses montrent que ce sont ces informations complémentaires qui apparaissent souvent déterminantes dans la décision évaluative de l'enseignant.

À partir de notre analyse des pratiques des enseignants, tentons de dégager le caractère social et éthique du jugement professionnel en évaluation. Dans quelle mesure s'inscrit-il dans des réseaux de communication et de significations partagées? Dans quelle mesure relève-t-il d'une "culture de l'évaluation" construite au sein des établissements scolaires? Quelles sont les valeurs et les stratégies pédagogiques liées aux décisions évaluatives? 


\section{Un processus de communication et les significations attribuées à la note}

Il est aujourd'hui reconnu que l'évaluation s'inscrit dans un processus de communication et de négociation, dont font partie les transactions de sens et les ajustements de rôles entre les acteurs (Hadji, 1999). Dans les pratiques décrites par les enseignants, cela s'observe à plusieurs niveaux:

- déjà dans le cadre de la restitution des résultats aux évaluations sommatives ponctuelles en cours de trimestre. Sur la base du travail réalisé, des interactions avec un élève ont lieu par exemple pour mieux comprendre ses erreurs ou mieux juger sa compréhension de la tâche. Ce faisant, l'enseignant introduit une dimension d'évaluation formative à la suite d'un contrôle écrit à visée sommative;

- dans le cadre des modalités de communication qui entourent la note du livret scolaire. Bien que ces modalités varient d'un enseignant à l'autre selon ses choix personnels, tous les enseignants de notre recherche s'engagent dans des échanges oraux avec les élèves au sujet des notes, des entretiens et autres rencontres avec les parents d'élèves, la formulation de commentaires écrits dans le livret, l'élaboration d'un dossier d'évaluation ou portfolio comprenant des travaux de l'élève.

Mais toute communication stipule un message (des messages) à transmettre. Un élément qui ressort de façon très marquante de nos analyses est la signification que l'enseignant confere à la note trimestrielle ou de fin d'année qu'il va attribuer au regard des enjeux d'apprentissage et d'orientation. En effet, la note, en tant que message, apparaît investie d'une fonction qui va au-delà d'une catégorie d'appréciation qui ne ferait que situer l'élève sur une «échelle de mesure» de 1 à 6. Lorsqu'ils hésitent entre les notes 3 et 4 , deux conceptions contrastées ressortent du discours des enseignants de notre recherche notamment par rapport aux mathématiques qui, rappelons-le, ont un rôle important dans l'orientation de l'élève vers les filières de l'enseignement secondaire:

- Certains enseignants, surtout en cours d'année, décident d'attribuer la note 3 (au lieu de 4) en considérant que cette note doit servir «d'avertissement»; ils cherchent ainsi à stimuler l'investissement de l'élève dans le travail scolaire et l'amener à améliorer ses résultats au dernier trimestre.

- D'autres enseignants optent pour la note supérieure (4 au lieu de 3), en la considérant comme une note "coup de pouce», car ils souhaitent soutenir et encourager l'élève dans une trajectoire d'apprentissage jugée globalement positive; ce type de décision intervient parfois en cours d'année mais surtout en fin d'année lorsque l'enseignant estime qu'il ne faut pas «barrer la route» à un élève qui serait capable de faire des études secondaires dans la filière prégymnasiale. 
Quel que soit leur choix, les enseignants disent qu'ils expliquent le sens de cette note à leurs interlocuteurs. Cette observation souligne la dimension sémiotique de la note, en tant que «signe» dans les processus de communication entre l'enseignant, l'élève et ses parents.

\section{Des pratiques concertées entre collègues: construction d'une culture de l'évaluation}

Dans nos entretiens de recherche, nous avons régulièrement demandé aux enseignants des éléments de justification de leurs pratiques d'évaluation. Tous les enseignants invoquent des concertations et des choix collégiaux pour justifier plusieurs aspects de leurs pratiques. Ces concertations se font soit avec un collègue en particulier, soit avec les enseignants d'une équipe de degré ou cycle ou encore avec les collègues de toute une école. Les enseignants disent qu'ils se mettent d'accord sur les contrôles écrits à soumettre aux élèves, sur le contenu des en-têtes aux évaluations (objectifs visés, critères), sur les modes d'appréciation (avec ou sans composantes quantitatives, les barèmes, etc.), sur le nombre de contrôles écrits par trimestre. Ainsi, le jugement professionnel de l'enseignant ne relève pas de choix individuels seulement, mais il s'inscrit dans une communauté de pratique, avec des décisions collectives, des outils construits avec d'autres collègues, des modes d'appréciation commune notamment. Des différences apparaissent toutefois dans le degré de concertation: les choix collégiaux rapportés par quelques enseignants portent sur l'ensemble du processus d'élaboration et de conception des contrôles écrits et des feuilles récapitulatives des résultats des élèves, alors que d'autres ne portent que sur certains aspects en particulier. On note que les enseignants parlent plus rarement de correction concertée des travaux des élèves. Le cadre de référence critériée et le barème ayant souvent été décidés ensemble pour les contrôles écrits d'une certaine importance, il ressort que la correction relève ensuite de la responsabilité et de l'autonomie de chaque enseignant. Il en va de même concernant la décision définitive d'attribution de la note trimestrielle et de fin d'année pour chaque élève.

Plusieurs enseignants soulignent l'importance d'offrir un système cohérent aux élèves et aux parents d'une même école par des pratiques d'évaluation communes. Pour eux, il faut aussi pouvoir les expliquer, comme par exemple le choix de ne pas calculer des moyennes (conformément aux directives officielles d'alors) afin d'avoir un espace pour des procédures souples et adaptées pouvant rendre compte des progressions d'apprentissage réalisées. Certains expliquent également que cela permet de limiter les «tactiques» bien connues des élèves qui dosent leurs efforts en fonction de l'évolution de leurs moyennes et de l'importance des disciplines dans les décisions de promotion et d'orientation (tactiques décrites par exemple par Chevallard, 1986).

Cette prégnance des choix collégiaux dans les discours des enseignants est cohérente avec le fonctionnement des écoles concernées par notre recherche qui accordent en général une importance particulière au travail en équipe. Ils peuvent 
être interprétés comme les signes d'une culture de responsabilité partagée à propos de l'évaluation des apprentissages des élèves (Legendre, 2001). Le jugement professionnel s'inscrit dans cette culture professionnelle qui est évidemment liée aux contraintes institutionnelles. Ainsi, les enseignants, lorsqu'ils justifient leurs pratiques, se réferent également aux directives et moyens officiels, par exemple en signalant des objectifs d'apprentissage définis dans les plans d'étude, la consigne d'insérer un en-tête aux contrôles écrits et l'élaboration de cet en-tête au regard de ce qui a été conseillé en formation continue, l'usage d'anciennes épreuves cantonales comme modèles pour construire d'autres outils d'évaluation.

\section{Des valeurs et des stratégies pédagogiques liées aux décisions évaluatives}

La plupart des enseignants interviewés accordent une grande importance à la cohérence entre ce qu'ils savent du travail des élèves en classe, perçu au travers de leurs interactions quotidiennes et leurs observations, et les résultats aux évaluations sommatives ponctuelles. Un autre point récurrent dans le discours des enseignants est leur volonté de valoriser l'investissement de l'élève dans le travail scolaire, sa participation active, les efforts fournis, la progression réalisée. Pour eux, il s'agit de prendre en compte dans le cadre d'informations autres que l'évaluation "papier-crayon", la "compréhension» que l'élève possède des concepts clé travaillés, telle que perçue dans le «vécu de la classe», dans le quotidien des activités d'enseignement/apprentissage et des interactions formatives qui ont lieu. Certains enseignants parlent de "valoriser les réussites» mises en regard avec les difficultés qu'ils connaissent de leurs élèves. Il s'agit d'aller "rechercher le positif»" pour le mettre "au bénéfice de l'élève», d'oser "parier sur son avenir». Pour le faire, tous semblent reconnaître la nécessité de s'appuyer sur des traces complémentaires aux contrôles écrits.

Ces quelques constats mettent en avant la limite que représentent les évaluations écrites ponctuelles pour apprécier les apprentissages des élèves et l'importance de pouvoir procéder à des ajustements évaluatifs qui permettent une certaine souplesse que les enseignants considèrent comme étant "au bénéfice de l'élève», qu'ils déploient "dans son intérêt». Ces valeurs s'observent dans certaines stratégies pédagogiques que les enseignants disent privilégier: le fait notamment de pondérer les évaluations sommatives par des appréciations qualitatives quand la situation l'exige de leur point de vue, de valoriser et d'encourager l'effort de l'élève, de prendre en compte les progressions d'apprentissage.

La tendance des enseignants à valoriser les réussites des élèves ne veut cependant pas dire une attribution de notes sans fondements. Ils soulignent la nécessité de "vérifier les intuitions» par la recherche de traces concordantes et discordantes; ils estiment qu'il faut "documenter les décisions» pour pouvoir les communiquer à autrui, l'élève, ses parents, des collègues. Plusieurs enseignants signalent la nécessité d'être dans une posture réflexive qui incite à se poser des questions sur ses choix, sur ses intentions pédagogiques et didactiques, à définir 
précisément les objectifs pédagogiques à atteindre, à «dire» les hésitations que l'on peut avoir quant à l'évaluation des apprentissages d'un élève, à reconnaître la complexité d'une situation donnée et ses incertitudes.

\section{Eléments de synthèse et de discussion}

Notre objectif, dans cet article, a été d'éclairer quelques caractéristiques du jugement évaluatif de l'enseignant lorsqu'il relève de sa professionnalité. Dans Allal et Mottier Lopez (2008), nous l'avons modélisé12 en quatre points principaux.

Les deux premiers points correspondent aux heuristiques qui sous-tendent les raisonnements des enseignants lors de leurs décisions d'attribution de notes dans le livret scolaire:

1) Le jugement professionnel en évaluation comprend deux démarches de base: réunir et confronter des sources d'information de nature diverse; les combiner dans un raisonnement interprétatif qui peut inclure un algorithme de calcul mais ne s'y réduit pas.

2) Il s'appuie sur des procédures d'évaluation que l'enseignant élabore et applique à tous les élèves, mais une de ses fonctions essentielles est d'assurer l'adaptation des procédures aux situations singulières qui l'exigent (pp. 225-226).

Nos analyses détaillées montrent que les décisions d'ajustements qualitatifs prennent sens par rapport au cursus de l'élève, à sa progression en cours d'année, à sa participation en classe, à sa situation familiale, mais également au regard des exigences et attentes, y compris implicites, du système scolaire.

Les troisième et quatrième points de notre modélisation soulignent la contextualisation du jugement de l'enseignant en matière d'évaluation:

3) Le jugement professionnel en évaluation est un acte cognitif individuel mais il s'élabore et sintègre dans un réseau de communication au sein d'un contexte social et institutionnel.

4) Le jugement professionnel en évaluation implique une mise en relation entre les exigences du système scolaire, la culture de l'établissement, les valeurs personnelles, les stratégies pédagogiques de l'enseignant (pp. 227-228).

En contraste avec les recherches qui traitent le jugement évaluatif comme un processus essentiellement individuel, consistant à apprécier les informations recueillies en vue de prendre une décision, nos analyses ont situé le jugement professionnel en évaluation dans un réseau de communication et d'échanges entre les différents partenaires concernés. Elles montrent que des significations sont attribuées aux notes, en plus de leur définition en termes de degré d'atteinte des objectifs. Ces significations jouent un rôle déterminant en cas d'incertitude, telle que l'hésitation entre deux notes.

Nous avons tout spécialement argumenté la multiréférentialité du jugement professionnel en évaluation, car s'ancrant dans des pratiques, des normes, des va- 
leurs, des significations rattachées à des contextes pluriels (la classe, l'établissement, l'institution scolaire). Les enseignants de notre recherche accordent une importance toute particulière à la communauté de pratique à laquelle ils participent, en termes de choix collégiaux et de culture de l'évaluation partagée au sein de l'établissement scolaire. Les enseignants ont exprimé des valeurs, qui reflètent souvent celles valorisées collectivement par la profession ${ }^{13}$, pour justifier les procédures d'évaluation mises en œuvre et les ajustements évaluatifs dont ils assument la responsabilité.

Alors, pour conclure, pourquoi considérons-nous qu'il est important de soutenir et de développer le jugement professionnel en évaluation? Qu'est-ce que cette conception apporte à l'évaluation des apprentissages? Quels sont les enjeux?

De nombreuses études ont souligné les biais qui peuvent intervenir dans les pratiques de notation des enseignants. Les plus connus, mis en évidence par les recherches docimologiques (e.g., Noizet \& Caverni, 1978; Piéron, 1963), sont par exemple les effets de halo (la note est «contaminée» par la connaissance d'autres caractéristiques de l'apprenant), d'assimilation (la note est influencée par les résultats antérieurs de l'élève), d'ordre et de contraste (la note est influencée par la qualité de la copie précédente). De notre point de vue, on doit s'interroger si tous ces «effets» sont du même ordre. Il est sans conteste injuste que l'ordre des copies ait une influence sur la note, tout comme certaines caractéristiques non scolaires de l'élève (son origine socio-économique, son sexe, son habillement, etc.). Par contre, la prise en compte par l'enseignant de certains facteurs tels l'investissement de l'élève dans le travail scolaire, le soutien familial, le souhait de l'élève et de sa famille pour l'étape suivante de la scolarité, etc. sont des éléments qui éclairent le travail scolaire et qui entrent alors dans le jugement professionnel en évaluation de l'enseignant. Si d'un point de vue métrique ces facteurs peuvent être considérés comme des biais, ils ont un autre statut par rapport au postulat d'éducabilité de l'élève, en tant que facteurs pertinents pour promouvoir sa progression scolaire et son développement personnel.

Des approches sociologiques ont, quant à elles, mis en évidence les différentes fonctions que l'évaluation peut jouer dans une classe, autres que pédagogiques et didactiques, amenant à des «arrangements» évaluatifs variés faisant l'objet de négociations implicites et explicites avec les élèves: par exemple, pour garantir l'ordre dans la classe, pour maîtriser les conflits maître-élèves, pour correspondre à la norme de notation dominante d'un établissement scolaire donné (Merle, 2007; Perrenoud, 1998).

Notre objectif n'est absolument pas de nier ces phénomènes. Comme discuté par Laveault (2005), les jugements de l'enseignant relèvent toujours d'aspects inconscients qui sont difficiles à contrôler. Les valeurs personnelles des enseignants ne sont pas forcément identiques et peuvent être plus ou moins en harmonie avec celles valorisées par le système scolaire et par la profession. Certaines croyances peuvent être inadaptées. Autrement dit, le jugement, y compris professionnel, relève toujours en partie d'aspects qui échappent partiellement à l'ac- 
teur (e.g., Bressoux \& Pansu, 2003), auxquels il n'est pas facile d'accéder, y compris par la pratique réflexive et la réflexion critique (et donc aussi dans le cadre d'entretiens de recherche). Toutefois, les recherches conduites auprès des enseignants primaires à Genève, ainsi que celles menées au Québec, mettent en évidence plusieurs démarches qui peuvent aider à limiter les biais évaluatifs et à améliorer la cohérence et la rigueur des pratiques de notation des enseignants (Lafortune \& Allal, 2008). Par exemple: expliciter les contenus évalués et la façon de les évaluer, travailler en équipe de professionnels et se concerter pour construire les outils d'évaluation, confronter les résultats de ses évaluations à ceux des évaluations externes et standardisées, enrichir ses interprétations au sujet des apprentissages d'un élève en sollicitant les avis d'autres professionnels.

Nous considérons que le fait de promouvoir le développement du jugement professionnel en évaluation est un moyen d'amener les enseignants à prendre conscience des biais évaluatifs et à adopter des démarches susceptibles de les réduire. Il s'agit de reconnaître que l'évaluation des apprentissages des élèves, sommative et pronostique, telle qu'étudiée dans notre recherche, implique des procédures rigoureuses, critériées, que l'enseignant élabore et applique à l'ensemble des élèves. Mais c'est également admettre qu'en cas de divergences entre les différentes informations recueillies (par exemple entre les résultats à un contrôle écrit et la réussite de l'élève en situation d'apprentissage), en cas d'hésitation entre deux notes, l'enseignant doit adapter ses procédures pour réexaminer les résultats et recueillir d'autres informations complémentaires. Ce faisant, c'est reconnaître la construction par l'enseignant d'une intelligibilité des situations; c'est donc s'éloigner de la vision d'un enseignant qui situerait mécaniquement l'élève sur une échelle de mesure à partir seulement de quelques contrôles écrits et autres interrogations ponctuelles. En acceptant la légitimité du jugement professionnel en évaluation, on admet une part de différenciation dans l'évaluation, qui se doit d'être objectivée par des démarches (individuelles et collectives) d'analyse réflexive et critique.

Evidemment, tous ces éléments impliquent un profond changement de la conception même de l'évaluation des apprentissages des élèves, de la part de l'ensemble des partenaires sociaux: enseignants, parents, autorités scolaires, responsables politiques. Le jugement professionnel en évaluation représente alors un objet crucial à aborder en formation initiale et continue, dans des projets de développement professionnel non seulement à titre individuel mais peut-être encore plus fondamentalement dans une perspective de concertation collégiale et de construction d'une culture de l'évaluation au sein de chaque établissement scolaire au regard de dispositions règlementaires qui soutiendraient cet aspect clé de la professionnalité de l'enseignant. 


\section{Notes}

1 Évidemment, la question découlant de cette affirmation est de savoir quels sont ces principes éthiques. Laveault en cite plusieurs, avec en surplomb la pensée philosophique d'Hadji (1997), à savoir: «n'exercer son pouvoir d'évaluateur que s'il contribue à la prise de pouvoir sur soi de l'évalué»...

2 Une recherche collaborative sur le jugement professionnel a été effectuée en parallèle au Québec et à Genève (Lafortune \& Allal, 2008). L'équipe responsable de l'étude genevoise, présentée dans cet article, était composée de chercheurs de l'Université de Genève (Linda Allal, Lucie Mottier Lopez, Walther Tessaro, Edith Wegmuller) et de membres du Centre de formation de l'enseignement primaire (Filippo Cattafi, Ariane Favre Marmet, JeanMarc Hohl, Bernard Riedweg). Nous remercions Jamila Dorner pour sa contribution à la récolte et au dépouillement des données, les enseignants qui ont participé aux entretiens pour leur apport précieux et la Direction de l'enseignement primaire du canton de Genève pour son soutien à cette recherche.

3 Voir Mottier Lopez (2008) pour une revue de littérature critique de cette perspective située dont une des thèses centrales est d'argumenter une relation de co-constitution entre activité et contexte.

4 Transformations successives conceptualisées par la notion bien connue de "transposition didactique».

5 Pour une présentation plus détaillée du contexte et du dispositif de la recherche, voir Allal, Wegmuller et Riedweg (2008).

6 Dans le système scolaire genevois, l'élève est orienté dès la $7^{\mathrm{e}}$ année de sa scolarité soit vers une filière prégymnasiale conduisant à l'enseignement secondaire supérieur et aux examens de maturité donnant accès aux universités suisses, soit à d'autres filières qui n'ouvrent pas sur la formation gymnasiale.

7 L'espace à disposition pour cet article de synthèse ne nous permet pas de présenter les multiples éléments - citations du discours des enseignants, extraits des commentaires inscrits dans les livrets, exemples des traces écrites et des documents évaluatifs établis par les enseignants - qui aideraient à étayer et à illustrer nos propos. Pour connaître ces éléments, le lecteur devra consulter les chapitres 7 à 9 du livre portant sur la recherche (Lafortune $\&$ Allal, 2008).

8 Nous regroupons par ce terme les différentes expressions utilisées par les enseignants, évaluations écrites, activités bilan, tests, contrôles, qui désignent toutes un instrument d'évaluation composé d'items écrits (questions, consignes, problèmes, etc.) auxquels l'élève doit répondre par écrit.

9 Principalement qualitatif, car cela ne signifie pas que certains enseignants ne comptent pas, par exemple, le nombre d'appréciations A (atteint) vs NA (non atteint). Mais ces indicateurs ne font pas l'objet d'opérations arithmétiques (pondérations, calculs de moyennes) comme c'est par contre le cas dans une logique quantitative.

10 Le décloisonnement implique l'organisation d'activités d'apprentissage destinées aux élèves de deux ou plusieurs classes, animées par les titulaires des classes concernées, parfois avec l'aide d'autres membres de l'école.

11 Aux séances de suivi collégial, les enseignants de toute l'école ou d'une équipe au sein de l'école se réunissent afin de discuter des situations d'élèves qui présentent des difficultés ou autres caractéristiques particulières, de confronter les observations et avis de différents enseignants et de réfléchir ensemble aux décisions à envisager pour soutenir les élèves dans leurs apprentissages.

12 La validité de notre modélisation doit évidemment être éprouvée par d'autres recherches, dans d'autres degrés et disciplines scolaires et dans d'autres lieux géographiques.

13 Les articles du code déontologique du Syndicat des enseignant(e)s romand(e)s qui constituent des repères pour l'exercice du jugement professionnel en matière d'évaluation sont cités dans Allal et al. (2008), p. 138. 


\section{Références bibliographiques}

Allal, L. (1988). Quantitative and qualitative components of teachers' evaluation strategies. Teaching and Teacher Education, 4, 41-51.

Allal, L. (2007). Evaluation dans le contexte de l'apprentissage situé: peut-on concevoir l'évaluation comme un acte de participation dans une communauté de pratiques? In M. Behrens (Éd.), La qualité en éducation: pour réfléchir à la formation de demain (pp. 39-56). Québec: Presses de l'Université du Québec.

Allal, L. (2008). Pratiques évaluatives des enseignants face aux méthodologies des recherches évaluatives sur le système scolaire. In L. Mottier Lopez \& M. Crahay (Ëd.), Évaluations en tension. Bruxelles: De Boeck.

Allal, L. \& Hohl, J.-M. (2008). Le rôle du jugement professionnel dans les adaptations et les modalités de communication de l'évaluation. In L. Lafortune \& L. Allal (Éd.), Jugement professionnel en évaluation: pratiques enseignantes au Québec et à Genève (pp. 187-201). Québec: Presses de l'Université du Québec.

Allal, L. \& Lafortune, L. (2008). À la recherche du jugement professionnel. In L. Lafortune $\&$ L. Allal (Éd.), Jugement professionnel en évaluation: pratiques enseignantes au Québec et à Genève (pp. 1-10). Québec: Presses de l'Université du Québec.

Allal, L. \& Mottier Lopez, L. (2008). Mieux comprendre le jugement professionnel en évaluation: apports et implications de l'étude genevoise. In L. Lafortune \& L. Allal (Éd.), Jugement professionnel en évaluation: pratiques enseignantes au Québec et à Genève (pp. 223239). Québec: Presses de l'Université du Québec.

Allal, L., Wegmuller, E. \& Riedweg, B. (2008). L'étude du jugement professionnel dans le contexte genevois: orientations conceptuelles et démarches de recherche. In L. Lafortune \& L. Allal (Éd.), Jugement professionnel en évaluation: pratiques enseignantes au Québec et à Genève (pp. 131-158). Québec: Presses de l'Université du Québec.

Bressoux, P. \& Pansu, P. (2003). Quand les enseignants jugent leurs élèves. Paris: Presses Universitaires de France.

Brown, J. S., Collins, A. \& Duguid, P. (1989). Situated cognition and the culture of learning. Educational Researcher, 18 (1), 32-42.

Chevallard, Y. (1986). Vers une analyse didactique des faits d'évaluation. In J.-M. De Ketele (Éd.), L'évaluation: approche descriptive ou prescriptive? (pp. 31-59). Bruxelles: De Boeck.

Gohier, C., Bednarz, N., Gaudreau, L., Pallascio, R. \& Parent, G. (Éd.). (1999). L'enseignant, un professionnel. Québec: Les Presses de l'Université du Québec.

Hadji, C. (1997). Pour une éthique de l'agir évaluationnel. Mesure et évaluation en éducation, $20(2), 7-26$.

Hadji, C. (1999). L'évaluation démystifiée. Paris: Éditions ESF.

Huberman, M. \& Miles, M. (1991). Analyse des données qualitatives. Bruxelles: De Boeck.

Lafortune, L. (2006). Exercice et développement du jugement professionnel, Accompagner l'évaluation des apprentissages dans l'école québécoise. Aide à l'apprentissage et reconnaissance des compétences, Fascicule 3, [Document inédit, MELS-UQTR]. Consulté le 27 juin 2007 dans www.uqtr.ca/accompagnement-recherche sous la rubrique "Accompagner l'évaluation des apprentissages dans l'école québécoise».

Lafortune, L. \& Allal, L. (Éd.). (2008). Jugement professionnel en évaluation: pratiques enseignantes au Québec et à Genève. Québec: Presses de l'Université du Québec.

Lave, J. \& Wenger, E. (1991). Situated learning: Legitimate peripheral participation. Cambridge: Cambridge University Press.

Laveault, D. (2005). Le jugement professionnel de l'enseignant: quel en est l'impact sur l'acte d'évaluer? Mesure et évaluation en éducation, 28 (2), 93-114.

Legendre, M.-F. (2001). Favoriser l'émergence de changements en matière d'évaluation. Vie pédagogique, 20, 15-19.

Merle, P. (2007). Les notes, secrets de fabrication. Paris: Presses Universitaires de France. 
Mottier Lopez, L. (2007). L'impact des communautés de pratique dans les entretiens de coévaluation. In A. Jorro (Éd.), Evaluation et développement professionnel (pp. 153-167). Paris: L'Harmattan.

Mottier Lopez, L. (2008). Apprentissage situé: la microculture de classe en mathématiques. Berne: Peter Lang.

Mottier Lopez, L. (à paraître). Reconnaissance d'une professionnalité: un processus situé d'évaluation interprétative. In A. Jorro (Éd.), Les défis de la reconnaissance professionnelle: évaluer, valoriser, légitimer. Ottawa: Presses Universitaires d'Ottawa.

Mottier Lopez, L \& Cattafi, F. (2008). Le processus du jugement professionnel comme fil conducteur dans l'attribution des notes. In L. Lafortune \& L. Allal (Éd.), Jugement professionnel en évaluation: pratiques enseignantes au Québec et à Genève (pp. 159-186). Québec: Presses de l'Université du Québec.

Noizet, G. \& Caverni, J.-P. (1978). Psychologie de l'évaluation scolaire. Paris: Presses Universitaires de France.

Paquay, L., Altet, M., Charlier, E. \& Perrenoud, P. (Éd.). (1996). Former des enseignants professionnels. Quelles stratégies? Quelles compétences? Bruxelles: De Boeck.

Perrenoud, P. (1998). L'évaluation des élèves. Paris: De Boeck.

Piéron, H. (1963). Examens et docimologie. Paris: Le Seuil.

Mots clés: jugement professionnel, notation, évaluation sommative, évaluation pronostique, école primaire

\section{Das professionelle Lehrerurteil in der Beurteilung: ein kognitiver Akt und eine umgesetzte soziale Praxis}

\section{Zusammenfassung}

Dieser Artikel untersucht die grundlegenden Merkmale über das Entstehen der professionellen Schülerbeurteilung. Konzeptionell wird davon ausgegangen, dass die Genese dieses Prozesses an kognitive Prozesse und an soziales Handeln geknüpft und an Kontextmerkmale gebunden ist. Vertreten wird eine professionelle Schülerbeurteilung, die auf einem multiplen Bezugssystem beruht, das Rahmenlehrpläne, Kompetenzraster und Bezugspunkte verschiedenster Art benötigt. Dieser Artikel beschreibt eine Forschungsarbeit, die mit zehn Lehrpersonen der 6. Klasse der Primarschule im Kanton Genf bezüglich ihrer summativen und prognostischen Schülerbeurteilung. Die Ergebnisse dieser Studie beschreiben das Vorgehen der Lehrperson bei der Setzung der Zeugnisnoten und ihrer Beurteilungsanpassungen, die sie individuums- und situationsspezifisch vornehmen. Besonders beachtet werden die sozialen und ethischen Dimensionen der Urteilsfindung. Ein Entwurf zur Modellierung dieses Prozesses in vier Punkten wird vorgestellt. Diese Modellierung kann als Grundlage zur Ausbildung und zur kollegialen Fortbildung dienen, um dem Prozess der professionellen Urteilsbildung eine innere Kohärenz zu verleihen und um fehlerbehaftete Verzerrungen bei der Notengebung einzudämmen.

Schlagworte: Schülerbeurteilung, Benotung, summative Beurteilung, prognostische Beurteilung, Grundschule 


\section{Il giudizio professionale nella valutazione: un atto cognitivo e una pratica sociale situata.}

\section{Riassunto}

L'articolo esamina le caratteristiche del giudizio professionale da parte degli insegnanti nella valutazione. Questo giudizio viene concettualizzato come un processo cognitivo e una pratica sociale legata a contesti differenti. La multireferenzialità del giudizio professionale nella valutazione viene discussa mostrando come vengano attivati dei quadri e dei punti di riferimento d'ordine differente. L'articolo presenta una ricerca sui processi di valutazione sommativa e prognostica messi in atto da una decina d'insegnanti ginevrini della 6a elementare. I risultati descrivono le procedure utilizzate per stabilire le note nel libretto scolastico e gli aggiustamenti valutativi effettuati in relazione a situazioni di allievi particolari. Vengono sottolineate le dimensioni sociali e etiche del giudizio e si abbozza una modellizzazione in quattro punti. Questa permette di fondare attività di formazione e di collaborazione collegiale atte a sviluppare la coerenza e il rigore del giudizio professionale degli insegnanti e a limitare così i condizionamenti che influiscono sulle loro pratiche di valutazione.

Parole chiave: giudizio professionale, nota, valutazione sommativa, valutazione pronostica.

\section{Professional judgement in assessment: a cognitive act and a situated social practice}

\section{Summary}

This article examines the characteristics of teachers' professional judgement in assessment, conceptualised as a cognitive process and as a social practice linked to several contexts. The multireferentiality of professional judgement in assessment is described and is shown to draw upon frameworks and references of different kinds. The article presents a study of the summative and predictive assessment procedures carried out by 10 sixth-grade teachers in Geneva. The results describe the procedures used by the teachers to determine the marks registered in the school report card and the assessment adjustments undertaken with respect to particular student situations. The social and ethical dimensions of professional judgement in assessment are emphasized. A four point model is proposed as a basis for training activities and collegial collaborations that can develop the coherence and rigor of teachers' professional judgement and thereby limit the biases affection their grading practices.

Key words: professional judgement, marking, summative assessment, predictive assessment, primary school 